ESJ Humanities

\title{
Genre et Acces au Foncier : Etude Comparative des Modes d'Acquisition de la Terre chez les Femmes du Sud et du Nord de la Cote d'Ivoire : Cas d'Akoupé et Becouéfin ; Nahoualakaha et Torgokaha
}

\author{
Affessi Adon Simon \\ Yapo Anthelme Kabié \\ Brou Gnangon Georgette \\ Université Peleforo Gon Coulibaly de Korhogo, Côte d'Ivoire
}

Doi:10.19044/esj.2022.v18n2p52

Submitted: 07 November 2021

Accepted: 13 January 2022

Published: 31 January 2022
Copyright 2022 Author(s)

Under Creative Commons BY-NC-ND

4.0 OPEN ACCESS

Cite As:

Affessi A.S., Yapo A.K. \& Brou G.G. (2022). Genre et Acces au Foncier : Etude Comparative des Modes d'Acquisition de la Terre chez les Femmes du Sud et du Nord de la Cote d'Ivoire : Cas d'Akoupé et Becouéfin ; Nahoualakaha et Torgokaha. European Scientific Journal, ESJ, 18 (2), 52. https://doi.org/10.19044/esj.2022.v18n2p52

\section{Résumé}

La présente étude s'est intéressée à la question du mode d'accès à la terre par les femmes du Nord, et celles Sud de la Côte d'Ivoire. Elle se propose de comparer les déterminants socioculturels de l'accessibilité différentiée à la terre chez les femmes Sénoufo et Akyé. La population ciblée pour l'étude menée entre Avril 2021 et Juin 2021 est constituée d'autorités coutumières, des leaders communautaires et des femmes résidentes ou non, et membres ou non des coopératives agricoles. La démarche sur laquelle s'est appuyée cette étude est de type qualitatif orientée selon la méthode comparative et conduite à l'aide d'entretien individuel, de focus groups et d'observation participante. La théorie comparative a été convoquée pour mieux faire la comparaison entre ces deux entités (femme du Nord et femme du Sud) et apporter un meilleur éclairage sur la question de leur accès à la terre. L'analyse de la question de l'accessibilité de la terre a montré que les femmes du Sud de la Côte d'Ivoire accèdent de plus en plus à la terre car les normes, règles et valeurs de cette société ont connu des mutations tendant à intégrer la femme dans le partage de la terre même si cela ne se fait pas de façon équitable entre l'homme et la femme. Chez les femmes du Nord de la Côte d'Ivoire, l'accès définitif à la 
terre est quasiment impossible à cause de la rigidité des valeurs, normes et règles qui régulent cette société.

Mots clés : Genre, accès à la terre, pesanteur, représentation, droit traditionnel Akyé, Sénoufo, Côte d'Ivoire

\title{
Gender and Land Ownership: A Comparative Study of Women's Modes of Land Acquisition in the South and North of Cote d'Ivoire: Case of Akoupe and Becouefin; Nahoualakaha and Torgokaha
}

\author{
Affessi Adon Simon \\ Yapo Anthelme Kabié \\ Brou Gnangon Georgette \\ Université Peleforo Gon Coulibaly de Korhogo, Côte d'Ivoire
}

\begin{abstract}
This study looked at the question of how women in the north and south of Côte d'Ivoire access land. It compares the socio-cultural determinants of differential access to land among Senoufo and Akye women. The target population is made up of the customary authorities, community leaders, and women who may or may not be residents or members of agricultural cooperatives. The study was conducted from April to June. The approach of this study was qualitative and comparative, using individual interviews, focus groups and participant observation. The comparative theory was used to better compare these two entities (women from the North and women from the South) and to shed more light on the issue of their access to land. The analysis of the issue of access to land showed that women in the south of Côte d'Ivoire are gaining access to land because the norms, rules and values of this society have changed to include women in the sharing of land, even if this is not done in an equitable manner between men and women. Among women in the north of Côte d'Ivoire, definitive access to land is almost impossible because of the rigidity of the values, norms and rules that regulate this society.
\end{abstract}

Keywords: Gender, access to land, gravity, representation, traditional Akyé law, Senoufo, Côte d'Ivoire

\section{Introduction}

Dans la plupart des pays africains, l'agriculture constitue l'essentiel de l'activité économique. Les femmes se sont impliquées dans l'agriculture afin 
de booster l'économie de leur nation, celles-ci constituant une grande partie de la population économiquement active. En tant qu'agricultrices et ouvrières agricoles, elles jouent un rôle crucial pour assurer la sécurité alimentaire des ménages (Boto, 2012). « Dans de nombreux pays, le rôle des femmes dans la production agricole a augmenté ces dernières années suite à la migration des hommes vers les zones urbaines. Elles effectuent la majorité des activités agricoles, détiennent le tiers de l'ensemble des entreprises et représentent, dans certains pays, jusqu'à $70 \%$ des employés » (Banque Africaine de Développement, 2015).

Au-delà de leurs activités génératrices de revenus, ces femmes sont les principaux leviers de l'économie domestique et du bien-être familial, et jouent un rôle absolument indispensable parfois méconnu des dirigeants au sein de leurs communautés et de leurs nations respectives (Banque Africaine de Développement, op.cit.). Malgré la présence de la femme dans toutes les activités agricoles ainsi que la fréquence de temps qu'elle passe dans les travaux champêtres, celle-ci dispose de droits très limités sur la terre pour la pratique de l'agriculture. Les femmes ivoiriennes du Sud et du Nord vivent la même réalité.

La présente étude se propose d'appréhender, dans une analyse socioanthropologique, les raisons de l'accessibilité différenciée à la terre entre les femmes du Sud et celles du Nord. Elle est subdivisée en deux grandes parties : la première traite de la gestion foncière au Nord et au Sud de la Côte d'Ivoire dans les localités de Nahoualakaha et Torgokaha, d'Akoupé et de Becouéfin ; la deuxième partie traite de modes d'accès à la terre au Sud et au Nord du territoire ivoirien. Dans une perspective comparative, il sera mis en évidence les représentations associées à l'accessibilité des acquéreuses de la terre dans le Nord et le Sud ivoirien ; il sera également déterminé les pesanteurs du droit coutumier foncier sur l'accessibilité différentiée à la terre des femmes Sénoufo et Akyé.

\section{Méthodologie}

\subsection{Sites d'étude}

L'étude sur le genre et le mode d'accès à la terre des femmes dans la société Akyé et Sénoufo s'est déroulée dans les régions de la Mé et du Poro. Quatre (4) sous-préfectures ont été visitées : au Nord, les sous-préfectures de Korhogo et de Tiorognaradougou dont les villages de Torgokaha et Nahoualakaha ont été visitées. Au Sud, les sous-préfectures d'Akoupé et de Becouéfin ont fait l'objet d'investigations pour la collecte des informations. Le choix de ces sous-préfectures et villages s'est opéré en tenant compte de la forte participation des femmes dans le milieu agricole et leur disponibilité à fournir les informations utiles pour la réalisation de ce travail. 


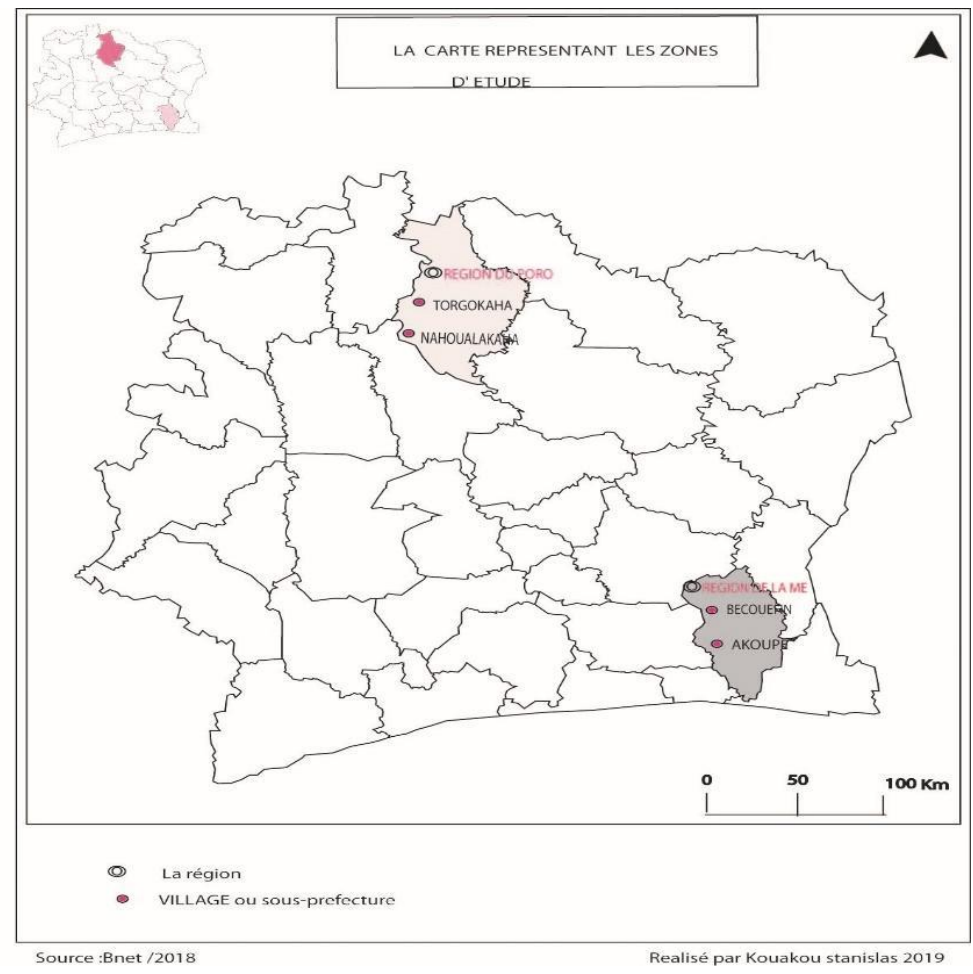

Figure 1 : Vue cartographique des zones de l'étude

Source : BNET, 2018

\subsection{Collecte des données}

La méthodologie a pour axe de référence scientifique le développement rural lié à la question du changement social. L'approche socioanthropologique permet de cerner les modes d'acquisition de la terre par les acteurs, en particulier les rapports entre les femmes et leur milieu social. Recherchant la qualité du discours des acteurs, la recherche a opté pour une démarche de type qualitatif, avec des techniques d'enquête comme l'observation participante, l'entretien semi-directif. Dans les quatre localités enquêtées, 54 acteurs ont été interrogés, et le principe de la saturation des informations a été observé. Ces acteurs sont répartis comme suit : 4 autorités villageoises, 4 présidents des jeunes, 4 chefs de famille, 6 femmes ménagères hors coopératives et 16 femmes des coopératives agricoles. Il s'agit des coopératives de COOPAB.COOP.CA ( 5 femmes), COOPAAS d'Akoupé (5 femmes) du Sud de la Côte d'Ivoire et des coopératives DJIDJA (5 femmes), WOWEDJO (5 femmes) au Nord ivoirien. En plus de ces entretiens, deux focus groups de 20 personnes ont été réalisées dans chaque localité avec les femmes acquéreuses ou en quête de terre pour la pratique d'activité agricole. Pour l'analyse des données, il a été procédé à la retranscription intégrale des entretiens réalisés avec les différents acteurs puis à une catégorisation des 
idées. À partir de cette retranscription, une analyse du contenu du discours des enquêtés a été réalisée.

\section{Résultats de l'étude}

\subsection{Gestion foncière et modes d'accès traditionnels}

\subsubsection{La gestion foncière au Nord et au Sud de la Côte d'Ivoire}

Comme dans la plupart des sociétés traditionnelles africaines, la gestion foncière est purement masculine. À Nahoualakaha et Torgokaha, cette donne est avérée ; la gestion de la terre est considérée comme une affaire des hommes, les femmes n'y interviennent pas. Dans ces villages, le terroir villageois est géré de concert par le chef du village et le chef de terre. Ils sont les garants du patrimoine foncier. L'attribution de la terre familiale ou communautaire à toute personne désireuse de terre pour une quelconque activité est du ressort des chefs de famille et les femmes ne sont pas convoquées. Certes, le chef de famille attribue les terres aux personnes désireuses, mais, le chef du village est informé de cette transmission de terre. Les femmes n'interviennent pas dans la gestion foncière, elles y accèdent par le biais des hommes. Monsieur S.K, chef de village de Torgokaha dit ceci :

«Chez nous ici, ce sont les hommes qui donnent la terre à celui qui veut. Ce sont les hommes qui donnent la terre aux femmes, les femmes ne peuvent pas donner la terre et c'est comme ça depuis longtemps».

Cette affirmation permet de comprendre que la gestion de la terre par les hommes ne date pas d'aujourd'hui, elle a pris forme depuis que le peuple sénoufo existe et ne peut pas être changée.

En ce qui concerne les villages d'Akoupé et Becouéfin, la gestion foncière est aussi du ressort des hommes et des femmes. Dans ces localités, la gestion foncière émane des chefs de famille. Bien qu'il existe des chefs de village et des chefs de terre, l'appropriation ou la distribution des terres est le devoir des chefs de famille; le chef du village n'intervient pas. De même, tout partage ou transfert de terre familiale ou communautaire se fait avec l'association des femmes. Les femmes font partie de la gestion du patrimoine foncier. L'implication de la femme dans la gestion de la terre est récente. Avant, cela n'était pas possible, les femmes n'étaient pas associées. Une enquêtée, A.N, responsable des femmes, affirme en ces mots :

"Dans notre village, c'est vrai que les hommes sont au-devant de la gestion de la terre mais ne peuvent pas donner la terre sans aviser les femmes, sinon ça va créer des palabres dans la famille».

On peut dire que pour le peuple Akyé, l'implication de la femme dans la gestion foncière permet de sauvegarder la cohésion dans la cellule familiale. 
Au Nord, les femmes ne sont pas associées dans la distribution et le partage des terres tandis qu'au Sud, elles sont de plus en plus avisées et font partie intégrante de cette gestion foncière. Dans le Nord, la gestion de la terre est vue comme une question de genre et le prolongement de la suprématie des hommes sur les femmes alors qu'au Sud la gestion de la terre est perçue comme le maintien de l'unité familiale et de la stabilité sociale.

\subsubsection{Modes d'accès à la terre au Sud et au Nord de la Côte d'Ivoire}

\subsubsection{L'héritage foncier}

L'héritage est le principal mode d'accès à la terre dans le village de Becouéfin et d'Akoupé. Dans la société Akyé, la succession à l'héritage est de type matrilinéaire, mais dans son application elle prend la forme patrilinéaire. En effet, dans les années 1960 jusqu'en 1990 lorsqu'un chef de famille décédait, le patrimoine foncier qu'il détenait était confié à son neveu utérin, c'est-à-dire au fills de la sœur aînée du défunt. Mais, aujourd'hui l'héritage foncier du défunt père est géré par ses enfants (filles et garçons). L'héritage a pris cette forme car aujourd'hui, lorsqu'un père décède, ce sont les enfants qui sont chargés de l'organisation des funérailles. Il arrive parfois que des filles qui ont un meilleur statut social prennent le devant des funérailles et gèrent toutes les dépenses. Ce qui fait que dans le partage du foncier, les femmes reçoivent aussi des portions de terre qu'elles exploitent à leur propre compte. A ce sujet, Dame A.B, de la coopérative COOPAB.COOP.CA de Becouéfin, dit ceci :

"Moi je n'ai pas eu la chance, j'ai perdu mon père et mon mari, mais ce qui est bon dans tout ça est que j'ai bénéficié de la terre de mon père et de mon mari lors du partage du foncier. Actuellement je travaille d'abord sur la terre de mon père et j'ai deux hectares de cacao que j'exploite pour moi-même. Quand je vais finir d'exploiter la terre de mon père, je vais attaquer pour mon mari ».

Le fait de permettre aux femmes d'avoir accès à la terre et de pratiquer l'agriculture ou les cultures de leur choix, permet à celles-ci d'être autonomes et de se réaliser. Il y a des femmes qui disposent de grandes constructions. Ces constructions sont les fruits de leur activité agricole. Dame Y.H, ménagère membre de coopérative, relève ce qui suit :

"Ma mère a eu un seul enfant et le seul enfant c'est moi. Après le décès de ma mère j'ai hérité de tous ses biens. À cela je dis merci aux frères de ma mère qui ne se sont pas opposés. J'ai hérité d'une plantation de cinq hectares, l'argent reçu de la récolte de cette plantation me permet de scolariser mon fils et d'aider mon mari. Comme mon fils a fini ses études, je suis en train de voir comment je vais faire pour construire ma maison». 
De même, s'il arrive qu'une femme soit omise lors de la distribution $\mathrm{du}$ foncier, cette dernière réclame sa part d'héritage. Parfois les femmes convoquent leur frère ou oncle chez le chef de famille ou du village pour réclamer leur part d'héritage foncier. On constate ainsi que les normes et les valeurs traditionnelles des sociétés Akyé sont plus flexibles dans la gestion de l'héritage foncier. Les propos d'O.J, de la coopérative COOPAAS d'Akoupé, nous permettent d'étayer cette idée :

"Après le décès de mon père, quand nous avons partagé la terre, il y avait un de mes grands frères qui voulait m'arracher ma place, il me disait que ma portion de terre était grande alors que je suis une fille et il a commencé à rentrer dans ma parcelle. Il disait aussi que les parents de mon mari ont assez de portions de terre et que mes enfants pourront bénéficier de ça. Je ne voulais pas alors je l'ai convoqué chez le chef du village. Le chef a pris ses notables pour aller voir et quand ils sont arrivés, ils ont constaté que j'avais raison. Ces derniers ont bien délimité la parcelle à l'aide d'un piquet et depuis ce jour-là je jouis de cette portion qu'il a voulu m'arracher $»$.

Contrairement aux femmes d'Akoupé et de Becouéfin, celles de Nahoualakaha et Torgokaha ont des difficultés dans l'accès à l'héritage foncier. Evoluant aussi dans un système matrilinéaire pur, l'héritage du père est confié à son neveu utérin, c'est-à-dire au fils de sa grande sœur. Cela s'explique par le fait que dans le fonctionnement de ce système, l'oncle maternel est le père réel (le père social légitime) de l'enfant. C'est l'oncle maternel qui s'occupe des neveux, les enfants n'héritent pas de leur père. À Nahoualakaha et Torgokaha, les femmes héritent exceptionnellement du patrimoine foncier, à condition qu'il n'y ait plus de descendant masculin dans la lignée ou la famille. Toutefois ce cas reste très rare. La plupart des femmes qui bénéficient de l'héritage foncier sont les femmes très âgées. Ces dernières lorsqu'elles gèrent le patrimoine foncier deviennent autonomes et arrivent à se réaliser comme celles d'Akoupé et Becouéfin. À Torgokaha, Monsieur S.T, un chef de famille dit :

"Moi-même qui vous parle, j'ai vécu ce cas. Après la mort de mon père ce sont ses neveux qui ont pris tout l'héritage, ils ont chassé ma mère et nous de la cour de notre père. La cour dans laquelle nous causons actuellement est ma propre cour. Je l'ai construite moi-même. Chez nous ici je ne parle pas d'ailleurs, les femmes héritent de la terre quand tous les hommes de leur famille sont décédés et que les garçons qui restent et qui doivent hériter sont encore petits ou sont des enfants $»$. 
Les propos de Madame O.N, présidente de la coopérative Djidja, viennent compléter celui de S.T :

"Nous les femmes on souffre trop, une fois tu perds ton mari on te chasse toi et tes enfants de la maison, car selon les sénoufo, c'est l'enfant qui va chercher son papa. Quand cela arrive tu ne peux pas aller te plaindre quelque part parce que c'est comme ça, ça se passe ici ».

Les propos des enquêtés permettent alors de dire que cette manière de faire émane des normes, des valeurs et des règles traditionnelles qui régulent la société sénoufo. Ces règles de par leur caractère rigide et transversal empiètent sur le droit des femmes et des enfants. Il faut aussi ajouter que plus les règles traditionnelles sont rigides moins les femmes ont accès à la terre pour le développement de l'agriculture.

On conclut pour dire, l'héritage est un moyen d'accès à la terre d'un côté et de l'autre une sorte de positionnement et du respect de statut lignager.

\subsubsection{La donation}

À Akoupé et Becouéfin, le don est un mode d'accès à la terre assez utilisé. Il se fait généralement entre les membres de la famille et même à un étranger en guise de reconnaissance de ses bienfaits. Dans cette forme de donation, on assiste au transfert des droits fonciers d'un propriétaire terrien à certains membres de sa famille. Cette donation se fait entre père et enfants (fils ou filles), entre mari et épouses, entre mère et enfants (fils ou filles), entre frères et sœurs et entre le chef de famille et une tierce personne qui peut être un étranger, à condition que ceux-ci aient la capacité de mettre en valeur la parcelle de terre qui leur a été attribuée.

D.A, membre de la coopérative COOPAB.COOP.CI, affirme:

"Mon père de son vivant $m$ 'avait donné une parcelle de terre devant tous mes frères. Cette parcelle je l'exploite jusqu'aujourd'hui. Mais après sa mort, j'ai reçu un peu de terre pendant le partage du patrimoine foncier ».

En ce qui concerne Nahoualakaha et Torgokaha, la donation de terre est très rare. Dans cette localité, la terre garde encore son caractère sacré. La terre est un bien familial, lorsqu'on la donne à autrui on perd totalement son droit de propriétaire et elle revient à un autre. Dans la société sénoufo connue comme société conservatrice, perdre son patrimoine foncier signifie perdre une partie de soi-même. Le mode d'accès des femmes le plus courant est le prêt de terre pour les besoins de subsistance. La terre ne peut être attribuée à un étranger sous forme de don dans cette localité comme on le fait au Sud. 
Si au Nord la donation de terre est quasiment absente dans le système de relation familiale et est considérée comme une perte de bien familial, au Sud par contre, elle prend la forme de reconnaissance d'un proche ou parent. Cela permet de resserrer les liens de parenté et de maintenir la cohésion familiale.

\subsubsection{Le prêt de terre}

Le prêt de terre pour les besoins de subsistance est le mode d'accès le plus utilisé dans le Nord en général, et à Nahoualakaha puis à Torgokaha en particulier. Principalement ce mode d'accès permet aux propriétaires terriens de garder toujours leur terre, car un prêt se fait à titre provisoire et périodique. Une fois la période d'occupation écoulée, le propriétaire reprend sa portion de terre et peut ensuite la prêter à un autre individu. On prête la terre à tout individu n'ayant pas assez de terre pour ses besoins de subsistance. Mais dans les clauses du contrat, il y a certaines cultures qui sont proscrites aux concessionnaires. Ces cultures sont entre autres l'anacarde, le coton, la mangue, en un mot les cultures pérennes. Ces cultures sont proscrites du fait qu'elles mobilisent le sol. Ainsi, le maraîchage demeure la forme la plus appropriée. C'est généralement dans ce mode d'accession à la terre que se trouvent les femmes de Torgokaha et de Nahoualakaha. Le prêt de terre y obéit à un processus traditionnel. En effet, les femmes demandeuses de terre se rendent chez le chef du village avec du bois de chauffe ou fagot. Ces dernières remettent le bois de chauffe au chef du village qui à son tour trouve un chef de famille afin qu'il prête une parcelle aux interessées. Les parcelles les plus couramment prêtées sont les bas-fonds. Sur ces terres, les femmes travaillent ensemble c'est-à-dire elles ont un champ communautaire, elles font uniquement les cultures vivrières (riz, maïs, patate etc.) et le maraîchage (oignon, tomate, choux etc.). Madame O.N, la présidente de la coopérative Djidja, livre :

"Nous on travaille dans un bas-fond, il appartient à une vieille femme qui a hérité la terre parce que dans sa famille tous les garçons sont morts et c'est elle seule qui est restée. Avant de nous prêter le basfond, nous avons donné du bois au chef du village. Il a intercédé auprès de la vieille femme qui a accepté de nous prêter le bas-fond. Ce qui est bon dans tout ça c'est que quand on récolte nos produits on ne donne rien à la femme parce qu'on a déjà donné du bois au chef du village. On fait seulement que du maraîchage et notre parcelle fait 2,5 ha».

Comme il a été mentionné un peu plus haut, le chef du village est le garant du patrimoine foncier. À Becouéfin et à Akoupé, le prêt de terre pour les besoins de subsistance n'est pas seulement l'affaire des femmes, car dans cette zone on prête la terre à tout individu n'ayant pas assez de terre pour ses 
besoins de subsistance. Les clauses de prêt de terre avoisinent celles établies à Nahoualakaha et Torgokaha c'est-à-dire qu'il y a certaines cultures qui sont proscrites aux concessionnaires. Ces cultures sont entre autres le cacao, le café, l'hévéa, le palmier à huile etc., en un mot les cultures pérennes. Ces cultures sont entre autre le cacao, le café, l'hévéa, le palmier à huile, etc., en un mot les cultures pérennes. Ces cultures sont proscrites du fait qu'elles mobilisent le sol. Ainsi, la culture vivrière demeure la forme la plus appropriée. Dans certaines clauses de prêt, le demandeur de terre a l'obligation de donner une partie de sa récolte au propriétaire terrien et dans ces conditions, les clauses sont établies sans l'intervention du chef du village et de façon orale. Le prêt de terre se fait à titre provisoire et périodique, une fois la période d'occupation écoulée, le propriétaire reprend sa portion de terre et il peut ensuite la prêter à un autre postulant. Cependant, les femmes des coopératives d'Akoupé et Becouéfin ne se trouvent pas dans ce mode d'accès à la terre. Elles ont chacune leur propre parcelle et ne travaillent pas ensemble, c'est-àdire qu'elles n'ont pas de champ communautaire. Parmi les femmes interrogées à Becouéfin dans la coopérative (COOPAB.COOP.CA), deux exploitent chacune une superficie de 05 hectares de café et de cacao, une autre exploite 2,5 hectares de cacao et café. Deux autres exploitent chacune 02 hectares de cacao, café et d'autres cultures telles que la banane, le manioc etc. Quant aux femmes d'Akoupé (COOPASS) quatre exploitent 03 hectares de café et cacao individuellement et une exploitent chacune 01 hectare de cacao et de café. Ces femmes ont presque toutes hérité des parcelles qu'elles exploitent de leur parent ou mari, à l'exception d'une seule qui a acheté sa terre sur fonds propres.

Cette disproportion de superficies culturales entre les femmes d'Akoupé, Becouéfin et de Torgokaha, Nahoualakaha montre que celles des coopératives (COOPAB.COOP.CA) et (COOPASS) du Sud possèdent plus de terres culturales que celles des coopératives WOWEDJO et DJIDJA du Nord bien qu'elles évoluent dans le même esprit coopératif. Il faut également noter qu'il y a une différenciation dans les clauses de prêt. D'un côté la présence du chef du village et de l'autre côté son absence. En clair, le prêt de terre est vu au Sud et au Nord comme une sécurisation et un maintien de ressources patrimoniales mais aussi comme un signe de générosité et d'altruisme envers les autres.

\subsubsection{La vente de terre}

Dans la culture Akyé, la terre avait un caractère sacré. elle était inaliénable car elle appartenait à la fois aux générations passées, présentes et futures ;; Celui qui l'exploite en tant que dépositaire doit user des fruits de cette terre pour prendre en charge les problèmes des vivants et morts. De nos jours, on assiste à une désacralisation de la terre, elle a perdu son caractère 
inaliénable et est devenu marchande. La vente est un autre mode d'accès à la terre pour le développement de l'agriculture. Au Sud, ce mode d'accès est légion dans la zone d'Akoupé et Becouéfin. Elle survient lors des difficultés familiales telles que le décès et les cas de maladie. Les hommes décident de vendre les terres familiales pour résoudre les problèmes. C'est à travers la vente de terre que certaines femmes accèdent à la terre. C'est le cas de Madame A.J de la coopérative d'Akoupé :

"Dans notre famille on n'a pas beaucoup de terre pour faire l'agriculture, alors j'ai fait un peu de commerce et quand j'ai eu un peu d'argent, j'ai acheté la terre d'un monsieur qui avait des difficultés et c'est cette terre qui me donne à manger aujourd 'hui car je fais toute sorte de cultures dessus».

Concernant, les femmes de Nahoualakaha et de Torgokaha, elles n'ont pas cette opportunité d'acheter la terre. Dans cette zone, la terre garde encore son caractère sacré, elle est inaliénable. Elle ne se vend pas mais se prête. La société Sénoufo conserve fermement encore le caractère sacré de la terre. L'idée selon laquelle la terre appartient à la fois aux générations passées, présentes et futures, celui qui l'exploite en tant que dépositaire doit, en plus de bénéficier des fruits de cette terre, prendre en charge les problèmes des vivants et des morts. Cela remet en cause l'accès à la terre des femmes. Dans l'idéologie des hommes en général, la femme dans un foyer est une tierce personne, elle n'est pas de la famille de l'époux. Alors, lui céder la terre est considéré comme une vente de terre qui ne sera plus profitable à la famille de l'époux qui risque de la perdre. Autrement dit, la famille de la femme gagne en patrimoine foncier tandis que du côté de son époux c'est une perte définitive $\mathrm{du}$ patrimoine ancestral. La terre est vendue quand elle est lotie. En ce moment, elle peut se vendre à toute personne. Monsieur S.K, chef de famille, déclare ceci :

"Chez nous ici on ne vend pas la terre aux personnes qui veulent faire des plantations, mais on vend les terrains lotis. Aujourd'hui presque tout l'espace qui entoure le village est loti et il y a des étrangers qui ont acheté aussi pas pour faire des activités agricoles mais pour construire des maisons ».

Au regard de ce qui précède, il peut être dit que dans la zone d'Akoupé et de Becouéfin il y a un effritement des règles culturelles sur le foncier, alors qu'à Torgokaha et Nahoualakaha les normes foncières gardent encore leur rigidité. En somme, la question de la vente des terres est considérée au Nord comme une désacralisation des normes valeurs sociales et foncières tandis qu'au Sud, elle est vue comme une aliénation, un bouleversement des règles institutionnelles et foncières. 


\section{Discussion}

La présente étude montre dans un premier temps que les femmes du Nord de la Côte d'Ivoire ont difficilement accès à la terre et cela est dû à la coercition et à la rigidité des hommes, des règles, des normes et valeurs qui régulent le champ social Sénoufo.

Dans un second temps, l'étude révèle que les femmes du Sud, contrairement à celles du Nord, ont un accès facile à la terre. En effet, cette accessibilité facile des femmes du Sud à la terre est le résultat de l'effritement et de la flexibilité des règles, valeurs, normes et des hommes qui veillent sur les institutions, et qui contrôlent l'arène sociale Akyé. Dans un contexte Africain, en Côte d'Ivoire, l'étude s'apparente à celle réalisée par (Koné \& Ibo, 2009), qui indexent la tradition comme étant à la base ou un élément favorisant les discriminations de genre, tout en confinant les femmes au rôle d'épouse, de mère, de nourrice et de main-d'œuvre familiale. En effet, quelle que soit l'aire géographique (Nord/Sud, savane/forêt), ethnoculturelle (senoufo/agni) ou sociale en Côte d'Ivoire, les femmes ont généralement des difficultés d'accès à la terre surtout pour la réalisation de cultures pérennes et pour l'obtention de revenus durables. Seulement $5 \%$ de femmes à Ferkessédougou et $30 \%$ dans la région d'Abengourou (50\% à Niablé) possèdent une plantation ou un verger.

De même, Gogoua (2015) aborde la question de la promotion et la protection des droits de la femme en Côte d'Ivoire, au niveau de l'excision, de la succession ou l'héritage et de l'accès à la terre. Pour elle, la propriété foncière en Côte d'Ivoire est régie par la loi sur le foncier $\mathrm{n}^{\circ} 98-750$ du 23 décembre 1998. Cette loi accorde l'égal accès à la terre aux femmes et aux hommes. Mais de fait, on note beaucoup d'abus et de discriminations à l'égard des femmes, situation fondée sur les croyances culturelles et traditionnelles.

Par ailleurs, au-delà de la Côte d'Ivoire, Gbêtoénonmon (2017) dénonce également la complexité d'accès des femmes à la terre au Benin. L'étude met en relief l'exclusion de la femme de l'héritage foncier au Sahel et en Afrique de 1'Ouest. Pour lui, malgré les avancées au plan juridique, la tradition et les pesanteurs sociologiques continuent de soumettre la femme (mère, fille et épouse) à de nombreuses discriminations en matière d'héritage foncier.

Aussi, Mateso (2017) montre-t-il que dans un pays comme le Nigeria, les femmes représentent environ 60 à $80 \%$ de la main-d'œuvre agricole. Pourtant, ce sont généralement les hommes qui prennent les décisions importantes concernant la gestion des exploitations. Les terres n'appartiennent qu'aux hommes. Les femmes peuvent seulement les cultiver. La loi coutumière les exclut totalement de la propriété foncière. Il affirme également que les femmes reconnaissent aussi que la terre étant une propriété collective qui se transmet de chef de famille à chef de famille, il est impossible pour une femme 
vivant en milieu rural au Mali de posséder des terres qu'elle peut exploiter à sa guise.

De plus, la FAO (2010) met en relief que les inégalités entre les sexes concernant les droits à la terre sont généralisées. Non seulement les femmes ont moins accès à la terre que les hommes, mais leur accès est aussi souvent restreint à ce qu'on appelle les droits fonciers secondaires, ce qui signifie qu'elles détiennent ces droits par les membres masculins de la famille. Les femmes risquent donc de perdre leurs droits en cas de divorce, de veuvage ou de la migration de leur mari. Les faits montrent aussi que les parcelles des femmes sont généralement de plus petite taille et de qualité inférieure. Cependant, certains auteurs évoquent une atténuation de la difficulté d'accès des femmes à la terre. Affessi et al (2017) dans leur étude montrent que le système «abouya-achi » a déconstruit les rapports entre les membres du lignage autour de la terre, rapports qui étaient autrefois basés sur la servitude de la gent féminine du fait de la tradition. Ce système « abouya-achi » a servi d'éveil de conscience aux femmes du milieu rural Akyé qui réclament de plus en plus leur droit de propriété foncière. Pour eux, cette nouvelle forme d'exploitation de la terre est donc un antidote pour corriger la discrimination faite à la femme depuis longtemps quant à son accès à la terre pour l'agriculture. Ainsi, ce nouveau mode d'accès (système abouya-achi) permet à la femme d'être propriétaire de plantations donc de la terre sur une durée indéterminée.

Dans ce même ordre d'idées, Kimani(2008) explique que, si par le passé, les femmes n'avaient pas de droits fonciers directs, elles étaient cependant protégées par des traditions qui leur permettaient de continuer à avoir accès aux terres même après une séparation, un divorce ou la mort de leur époux. Il existait également des moyens d'arbitrage traditionnels auxquels les femmes pouvaient avoir recours si l'accès aux terres leur était refusé. Ces études s'apparentent à un volet de la présente qui montre que les femmes du Sud ont de plus en plus accès à la terre.

De façon pratique on retient que le problème de l'accès des femmes à la terre est favorisé dans la plupart des cas par les valeurs, normes et règles traditionnelles qui régulent le milieu rural et nos sociétés.

\section{Conclusion}

La comparaison des modes d'acquisition de la terre chez les femmes Sénoufo et Akyé a permis d'une part, de connaître les représentations associées à l'accessibilité des acquéreuses de la terre dans le Nord et le Sud ivoirien, et d'autre part, de déterminer les pesanteurs du droit coutumier foncier sur l'accessibilité différentielle à la terre chez les femmes Sénoufo et Akyé. Alors si les femmes d'Akoupé et de Becouéfin au Sud ont un accès plus facile à terre et sur les terres qu'elles ont à leur possession, elles pratiquent les 
cultures pérennes et vivrières ; c'est parce que dans ces localités, céder la terre à une femme est un moyen d'union et de consolidation des liens de parenté.

Cependant, à Nahoualakaha et à Torgokaha au Nord, les femmes ont difficilement accès à la terre ; et sur les parcelles qui leur sont octroyées, elles font uniquement les cultures saisonnières ou vivrières. Dans la perception Sénoufo, céder la terre à une femme signifie l'attribuer à une personne étrangère, à autrui ; ce qui prend aussi le sens de perte de patrimoine ancestral et de soi-même.

Alors on peut affirmer qu'au Nord, l'acquisition de la terre par les femmes est complexe du fait que dans cette aire culturelle, les valeurs et règles traditionnelles gardent encore leur caractère rigide et coercitif. À l'inverse, au Sud il y a une flexibilité dans l'acquisition de la terre par les femmes tout simplement parce que dans cette aire culturelle, on observe un décloisonnement des normes et valeurs traditionnels.

\section{References:}

1. AFFESSI, Adon Simon (2012). Innovations technologiques et participation paysanne au développement rural : L'exemple de l'ANADER dans le pays akyé. Thèse unique de doctorat, Université Félix Houphouët Boigny de Cocody, Abidjan. Institut d'EthnoSociologie, 354p.

2. AFFESSI Adon Simon, VANGA Adja Ferdinand \& GACHA FranckGautier (2017). Genre et dynamique des modes d'accès aux ressources foncières en côte d'ivoire : persistance ou début d'une indépendance socio-foncière des femmes de la société akyé ?, Institut National de la Recherche Scientifique (INRS), Lomé - Togo, Vol 11, n² - ISSN 0531 - 2051. 42-56.

3. Banque Africaine de Développement (2015). Autonomiser les femmes africaines: Plan d'action, Groupe de la Banque africaine de développement, Indice de l'égalité du genre en Afrique. Publié par Département du contrôle de la qualité et des résultats, Présence africaine, $17 \mathrm{p}$.

4. BOTO Isolina (2009). Accès à la terre et développement rural : nouveaux enjeux, nouvelles opportunités. Technical Centre for Agricultural and Rural Coopération ACP-UE (CTA), 45p.

5. DIAN Bony(1970). Le pays Akyé : Etude de l'économie agricole : in Annales de l'Université d'Abidjan série G, 206 p.

6. FAO(2010). Genre et droit à la terre. Département du Développement Économique et Social, Organisation des Nations Unies pour l'alimentation et l'agriculture, Viale delle Terme di Caracalla, 00153 Rome, Italie, 2p. 
7. GBETOENONMONA. (2020). Bénin : 05 raisons pour laisser les femmes accéder à la terre en sécurité, fondation Konrad-Adenauer/ WiLDAF/FeDDAF-Bénin/, AFRIQUE FONCIER $\mathrm{N}^{\circ} 003$ 4ème TRIMESTRE 2020, 23-26.

8. GOGOUA Rachel(2015). Thème : "Les traditions et coutumes qui empêchent l'égalité entre hommes et femmes ", 59ème Session CSW, New York du $x$ aux mars.

9. KONE Mariatou \& IBO Guehi Jonas, (2009). les politiques foncières et l'accès des femmes à la terre en côte d'ivoire : cas d'affalikro et djangobo (est) dans la région d'abengourou et de kalakala et togogniere (nord) dans la région de ferkessedougou,Femmes et foncier, Comité technique «Foncier \& développement », ministère des affaires Etrangères et européennes, Abidjan, Les Editions du CERAP/NEI.

10. KONE Mariatou (2006). Foncier rural, citoyenneté et cohésion sociale en Côte d'Ivoire : la pratique du tutorat dans la sous-préfecture de Gboguhé. Colloque sur Les Frontières de la question foncière : enchâssement social des droits et politiques publiques. Montpellier, 17,18 et 19 mai.

11. MARY Kimani (2008). Droits foncier: Le combat des femmes, Agriculture et luttes pour la terre, Afrique terre, Afrique renouveau, $5 \mathrm{p}$.

12. SEKA Aman Justin (2003). Approche socio-anthropologique de la gestion du patrimoine forestier en Côte d'Ivoire. Thèse de troisième cycle en sociologie non publiée, Abidjan Université Cocody. 